\title{
Astrometric Detection of Intermediate-Mass Black Holes At the Galactic Centre
}

\author{
Eden Girma, ${ }^{1 \star}$ and Abraham Loeb ${ }^{1}$ \\ ${ }^{1}$ Department of Astronomy, Harvard University, 60 Garden St., Cambridge, MA 02138
}

Accepted 2018 September 21. Received 2018 September 14; in original form 2018 July 6

\begin{abstract}
We assess the astrometric detectability of intermediate-mass black holes populating the inner parsec of the Milky Way Galaxy. The presence of these objects induces dynamical effects on Sgr A* and the star S2, which could be detected by next generation astrometric instruments that enable micro-arcsecond astrometry. An allowed population of ten $10^{4} M_{\odot}$ IMBHs within one parsec induces an angular shift of $\sim 65 \mu$ as $\mathrm{yr}^{-1}$ on the position of Sgr A*, corresponding to a perpendicular velocity component magnitude of $1.6 \mathrm{~km} \mathrm{~s}^{-1}$. It also induces changes in the orbit of S2 that surpass those induced by general relativity but lie within observational constraints, generating a mean angular shift in periapse and apoapse of $62 \mu$ as and $970 \mu$ as respectively.
\end{abstract}

Key words: black hole physics - methods: numerical - galaxy: centre

\section{INTRODUCTION}

The mass distribution of detected black holes exhibits an absence of intermediate-mass black holes (IMBHs), with masses of $10^{2}-10^{5} M_{\odot}$. These black holes are too massive to form from the collapse of present-day stars, their dynamical effect are too weak to be easily detectable, and their supposed environments lack the high density and velocities that could potentially fuel growth. Despite the relatively weak observational evidence available, IMBHs continue to represent a critical missing link between stellar and supermassive black holes, and a definitive discovery could provide essential insights into black hole formation, evolution, and dynamics.

There is a range of models for IMBH formation, including the direct collapse of very massive Population III stars (Bromm et al. 1999; Abel et al. 2000; Figer et al. 1996), gas accretion onto stellar-mass black holes (Leigh et al. 2013), and stochastic formation via black hole mergers and mass transfers in black hole binaries Giersz et al. (2016). Dense stellar clusters are also proposed to form IMBHs through the collapse of massive runaway mergers (Ebisuzaki et al. 2001; Zwart \& McMillan 2002); though such mergers are likely to experience severe mass loss though stellar winds, collapsing to black holes of mass $\lesssim 250 M_{\odot}$ (Petts \& Gualandris 2017). These dense clusters are characterised by a relaxation time, $\tau_{r}$, during which a test object changes its velocity by an order itself due to random perturbations induced by surrounding

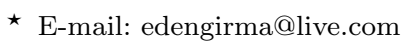

gravitational fields (Chandrasekhar 1941),

$\tau_{r} \sim \frac{\sigma^{3}}{8 \pi G^{2} \mu^{2} n \ln \Lambda}$

where $\mu$ is stellar mass, $\sigma$ is the velocity dispersion, $n$ is the number of stars per unit volume, and $\Lambda$ is the ratio of the maximal and minimal impact parameters, $b_{\max }$ and $b_{\text {min }}$. For a virialised and self-gravitating system with average stellar mass $\left\langle m_{*}\right\rangle$, the typical $b_{\min }$ (which leads to a deflection angle of $\pi / 2)$ is $G\left\langle m_{*}\right\rangle / \sigma^{2}$, and $b_{\max }$ is on the order of the half-mass radius $R_{h}$ (Freitag et al. 2006). A larger mass object $M$, travelling with velocity $v_{M}$ and embedded in spherically symmetric distribution of lower-mass objects $m$ with velocities $v_{m}<v_{M}$, will experience a decelerating force characterised by the Chandrasekhar dynamical friction formula

$\frac{d \vec{v}_{M}}{d t}=-16 \pi^{2} \ln \Lambda G^{2} m(M+m) \frac{\int_{0}^{v_{M}} f(r, v) v^{2} d v}{v_{M}^{3}} \vec{v}_{m}$

inducing migration of this larger mass to the centre of the system over time. In this vein, globular clusters of mass $M$ orbiting a host galaxy centre with circular velocity $v_{c}$ eventually spiral inwards from an initial radius $r_{i}$ after a time (Binney \& Tremaine 2011, eq. 7-25)

$$
\begin{aligned}
t_{\text {fric }} & =\frac{1.17}{\ln \Lambda} \frac{r_{i}^{2} v_{c}}{G M}=2.64 \times 10^{10} \mathrm{yr}\left(\frac{\ln \Lambda}{10}\right)^{-1}\left(\frac{r_{i}}{2 \mathrm{kpc}}\right)^{2} \times \\
& \times\left(\frac{v_{c}}{250 \mathrm{~km} \mathrm{~s}^{-1}}\right)\left(\frac{M}{10^{6} M_{\odot}}\right)^{-1},
\end{aligned}
$$

theoretically populating the centre of the Milky Way with stars, stellar remnants, and IMBHs. 
Supposing the existence of a surrounding IMBH population in the Galactic Centre, dynamical friction is believed to segregate these higher mass objects into a density cusp proportional to $r^{-7 / 4}$ (Morris 1993). These processes of relaxation and mass segregation have not only been demonstrated by various $N$-body codes simulating the dynamics of IMBHs in dense or in-spiralling stellar clusters (see Zwart \& McMillan 2002; Gürkan et al. 2004; Antonini et al. 2012; Mastrobuono-Battisti et al. 2014), but may also provide a necessary explanation for the perplexing number of massive main-sequence stars present around the centre of the Milky Way (the so-called "paradox of youth"; see Ghez et al. 2003; Paumard et al. 2006; Ghez et al. 2008; Lu et al. 2008; Yelda et al. 2014). The orbital properties of these young stars reflect what would result from millions of years' interaction with an IMBH of mass $\gtrsim 1500 M_{\odot}$, further supporting an IMBH transfer model (Merritt et al. 2009; Fujii et al. 2009, 2010). Additional models for young stellar populations in the Galactic Centre suggest in-situ formation (Bonnell \& Rice 2008; Mapelli et al. 2008; Alig et al. 2011, 2013) or tidal disruption of stellar binaries (Miller et al. 2005; Bromley et al. 2006; Perets et al. 2007, 2009).

The central supermassive black hole of the Milky Way (Sgr $A^{*}$ ) will experience reflex motion due to gravitational perturbations, which may be distinguishable in magnitude from changes induced by surrounding stars (Merritt et al. 2007; Chatterjee et al. 2002; Loeb \& Furlanetto 2013; Hansen \& Milosavljević 2003; Yu \& Tremaine 2003). The star S2, which orbits Sgr A* with a semi-major axis of $\sim 5$ milliparsecs (mpc) (Gillessen et al. 2009), might also hint at the existence of IMBHs through changes in eccentricity and orbital plane that surpass effects of general relativity (Gualandris et al. 2010; Weinberg et al. 2005). Hence, highaccuracy measurements of residual proper motion of Sgr A* so far quantified by Reid \& Brunthaler (2004) and evolving orbital parameters of $\mathrm{S} 2$ are essential in helping discern whether the inner parsec hosts IMBHs.

In what follows, we investigate whether particular IMBH populations in the Galactic Centre, characterised by various density profiles and black hole masses, produce a gravitational effect on Sgr $\mathrm{A}^{*}$ and $\mathrm{S} 2$ that is both detectable astrometrically and differentiable from the effects of surrounding stars. Using model density profiles for stellar- and intermediate-mass black holes (Section 3), we developed a code that used the open-source $N$-body integrator REBOUND (Rein \& Liu 2012) to create a simulated orbital system involving Sgr A*, a surrounding population of black holes, and the star S2 (Section 2). S2 is analysed over other S-stars, as it is one of the brightest of its counterparts and possesses an orbital period within 16 years. With this, its observed perturbations are particularly useful in characterising the surrounding gravitational potential. Note that the effects of IMBH perturbations are necessarily expected on other Sstars, and our code is made adaptable such that one could model the effects of an IMBH population on an S-star of one's choosing.

By simulating scenarios in which the central parsec is populated or not populated with black holes, we identify in Section 4 the dynamical signature of the IMBHs. Section 5 summarises our findings in the context of current observational constraints, and discusses how they may inform fu- ture observations by the Event Horizon Telescope $(\mathrm{EHT})^{1}$ and GRAVITY ${ }^{2}$ instrument on the VLT data collections on the angular displacements of Sgr A* and the orbit of S2.

\section{NUMERICAL METHODS}

To simulate the various $N$-body models involving Sgr $\mathrm{A}^{*}$, $\mathrm{S} 2$, and a surrounding population, we developed a Python package using the open-source, multi-purpose $N$-body software package REBOUND (Rein \& Liu 2012). REBOUND is designed to integrate a variety of gravitational systems and supporting both collisional and collision-less (classical) dynamics; upon importing the package, simulations may be initialised and performed via a provisional Python module. The Python package created for the purposes of this work used REBOUND to randomly realise and integrate two types of orbital systems: (i) Sgr A* and S2 along with varied IMBH and stellar populations, and (ii) Sgr $\mathrm{A}^{*}$ and S2 affected by a smooth density profiles matching those of the examined surrounding populations. These systems were differentiated to properly investigate how the effects of discrete objects on the orbital parameters of S2 differ from that of a smoothly distributed mass.

Each simulation is initialised with two active particles: a central black hole of mass $M_{\mathrm{cbh}}=4.4 \times 10^{6} M_{\odot}$ resting at the origin, and a star of mass $20 M_{\odot}$ representing S2, with orbital parameters provided by Gillessen et al. (2009). To add particles of mass $m$ reflecting a density profile $\rho$ that extends within a narrow range from a distance $r_{\min }$ to $r_{\max }$, we first calculate the number of particles present in the simulation $N_{\mathrm{p}}$. This is obtained by drawing randomly from a Poisson distribution with mean

$N_{\text {mean }}=\frac{1}{m}\left(M\left(r_{\max }\right)-M\left(r_{\min }\right)\right)$,

where

$M(r)=\int_{0}^{r} 4 \pi s^{2} \rho(s) d s$

describes the total mass contained within a radius $r$ for a density profile $\rho$. To distribute these particles according to their density profile, we randomly determine the distance of each particle from the origin through an inverse transform sampling method. The appropriate cumulative density function for particle position is

$f(r)=M(r) /\left(M_{\mathrm{c}}\right)$

where $M_{\mathrm{c}}$ is the total mass contained in the density profile $\rho$. We uniformly draw a value $u$ from the interval $\left(f\left(r_{\min }\right), f\left(r_{\max }\right)\right) \subset[0,1]$, let $m_{u}=M_{\mathrm{c}} * u$, and calculate the particle position as $r=f^{-1}\left(m_{u}\right)$. The unit directional vector $\hat{\mathbf{r}}=(x, y, z)$ of the particle position is then determined via sphere point picking: given random variate $u \in[-1,1]$ and $\theta \in[0,2 \pi)$,

$x=\sqrt{1-u^{2}} \cos \theta$

$y=\sqrt{1-u^{2}} \sin \theta$,

$z=u$.

1 https://eventhorizontelescope.org/

2 http://www.mpe.mpg.de/ir/gravity/ 


\begin{tabular}{lccccc}
\hline & $\left\langle m_{\rho}\right\rangle=1 M_{\odot}$ & $\left\langle m_{\rho}\right\rangle=10 M_{\odot}$ & $\left\langle m_{\rho}\right\rangle=100 M_{\odot}$ & $\left\langle m_{\rho}\right\rangle=10^{3} M_{\odot}$ & $\left\langle m_{\rho}\right\rangle=10^{4} M_{\odot}$ \\
\hline $\begin{array}{ccccc}\text { Error threshold } \\
\text { Discrete }\end{array}$ & $10^{-4}$ & $10^{-7}$ & $10^{-7}$ & $10^{-7}$ & $10^{-7}$ \\
Smooth & $10^{-4}$ & $10^{-5}$ & $10^{-5}$ & $10^{-4}$ & $5 \times 10^{-3}$ \\
$\frac{N_{\text {runs successful }}}{N_{\text {particles }}}$ & 309 & 413 & 1299 & 1394 & 943 \\
Mean & 31922 & 11462 & 358 & & \\
Max & $32069[0.3 \%]$ & $11623[0.2 \%]$ & $405[0.1 \%]$ & $5[17.8 \%]$ & $14[1.5 \%]$ \\
Min & $31770[0.3 \%]$ & $11272[0.2 \%]$ & $321[0.1 \%]$ & $0[0.1 \%]$ & $5[0.5 \%]$ \\
\hline
\end{tabular}

Table 1. The error threshold determining which simulations of discrete and smooth density profiles were analysed, number of successful simulation runs (out of 1500), and the average, minimum, and maximum number of particles drawn from the tested density profiles. Bracketed percentages indicate the percentage of runs that involved the maximum or minimum number of particles.

The phase space distribution of the orbital system is used to randomly determine the velocity of each particle at a known position $\mathbf{r}$. This distribution function is approximated as the analytic solution found by Tremaine et al. (1994) that corresponds to a Hernquist profile with a central black hole:

$$
f(\mathcal{E})=\frac{2 \Gamma(2)}{2^{7 / 2} \pi^{5 / 2}\left(G M_{\mathrm{c}} a\right)^{3 / 2} \mu \Gamma\left(\frac{1}{2}\right)} \mathcal{E}^{-1 / 2} .
$$

where $M_{\mathrm{c}}$ is the total cluster mass, $\mu$ is the ratio of central black hole mass to cluster mass, and $\Gamma$ the gamma function. Within a sufficiently thin spherical shell the phase space distribution depends only on velocity. Hence from Equation (9), we inversely derived continuous density functions describing the distribution of bound velocities $(v<\sqrt{-2 \Phi})$ for $10^{3}$ spherical shells of thickness $10^{-3} r_{\max }$ situated between $r_{\min }$ and $r_{\max }$. The speed of a particle at position $\mathbf{r}$ is randomly drawn from the continuous density function in the corresponding spherical shell. Upon drawing this magnitude, the direction of the velocity vector is randomly determined and ensured to be perpendicular to the position vector. The particles are then added to the simulation, specified by the orbital elements calculated from their position and velocity vectors, such that they are gravitationally interacting with the central massive object and the S-star, but not with each other.

For simulations testing the effects of smooth density profiles, we do not add any other particles besides Sgr A* and S2. Instead, we include an additional force induced by the smooth density profile in question.

For each density profile, we performed 1500 different random realisations of the associated particle system surrounding Sgr A* and S2. $N$-body simulations were advanced using the hybrid symplectic integrator Mercurius, which combines the symplectic WHFast and implicit IAS15 integrator modules already built into REBOUND (Rein \& Spiegel 2014; Rein \& Tamayo 2015). The properties of the integrator were further set such that the simulation merges particles that collide with each other, preserving mass and momentum, and tracks energy that is lost due to collisions. Error is kept at a minimum by identifying the optimal time step for each simulated scenario, which ranged from 20 hours to 80 hours. Between each call of the integration method, we record the position and velocity Cartesian components of $\mathrm{Sgr} \mathrm{A}^{*}$ and $\mathrm{S} 2$, the various orbital elements of $\mathrm{S} 2(a, e, i, \Omega$, and $\omega)$, and the total number of particles remaining in the simulation. Relative energy error was calculated, and simulations reaching energy errors greater than $10^{-7}\left(1 \times 10^{-4}\right.$ for stellar control) were discarded from the analysed data-set. The number of successful runs, along with the average, maximum, and minimum number of particles for each simulated model are additionally summarised in Table 1.

\section{THEORETICAL MODELS}

The numerical simulations described in Section 2 examined five distinct density profiles: a stellar control, stellar-mass black holes of $10 M_{\odot}$ and $100 M_{\odot}$, and intermediate-mass black holes of $10^{3} M_{\odot}$ and $10^{4} M_{\odot}$. Note that although we choose to include the IMBH mass of $10^{4} M_{\odot}$ in this work, IMBHs of mass $\gtrsim 10^{4} M_{\odot}$ have been largely excluded from most parameters in the Galactic Centre (see Reid \& Brunthaler 2004; Gualandris \& Merritt 2009).

The stellar control is described by a Hernquist (1990) spherical density profile

$\rho_{\mathrm{H}}(r)=\frac{M_{\mathrm{c}}}{2 \pi} \frac{a}{r(r+a)^{3}}$

where $M_{\mathrm{c}}=4.4 \times 10^{6} M_{\odot}$ is the total cluster mass, and $a=$ $1 \mathrm{pc}$ the scale length. We adopt this profile as a relatively close approximation to the actual broken law stellar density in the inner parsec, provided by Schödel et al. (2007) as

$\rho_{*}(r) \simeq(1.7 \pm 0.8) \times 10^{6}\left(\frac{r}{0.22 \mathrm{pc}}\right)^{-\gamma} M_{\odot} \mathrm{pc}^{-3}$,

where $\gamma=1.2$ for $r<0.22 \mathrm{pc}$ and $\gamma=1.75$ for $r>0.22 \mathrm{pc}$. We assume a stellar characteristic mass and radius of $1 M_{\odot}$ and $1 R_{\odot}$ respectively. The inner cut off radius for this profile is set as the tidal radius,

$$
\begin{aligned}
r_{t} & \simeq R_{\star}\left(M_{\mathrm{BH}} / M_{\star}\right)^{1 / 3} \\
& =0.76 \mathrm{AU}\left(\frac{R_{\star}}{R_{\odot}}\right)\left(\frac{M_{\mathrm{BH}}}{4.4 \times 10^{6} M_{\odot}}\right)^{1 / 3}\left(\frac{M_{\star}}{M_{\odot}}\right)^{-1 / 3} .
\end{aligned}
$$

The displacement of Sgr A* over year-long timescales is dominated by components orbiting around $0.01 \mathrm{pc}$, with analytic treatments finding proper motions of $\sim 0.07 \mathrm{~km} \mathrm{~s}^{-1}$ when considering stars within 2 pc of the Galactic Centre. (Broderick et al. 2011; Reid \& Brunthaler 2004). Thus, to shorten computation time while maintaining reasonable accuracy on our results, we simulate the stellar density profile out to 0.1 pc.

Density profiles for stellar-mass black holes are adapted from the methods of Miralda-Escudé \& Gould (2000), which 
construct a density profile of stellar remnants that is necessarily a fraction of that of the surrounding stellar cluster: for a characteristic black hole mass $M_{\mathrm{BH}}$ and stellar density profile $\rho_{*}(r)$,

$\rho_{\mathrm{SBH}}(r)=C \rho_{*}(r), C \equiv\left(\frac{0.23 M_{\odot}}{\left\langle M_{\mathrm{BH}}\right\rangle}\right)^{\frac{1}{2}}$,

where $M_{\mathrm{BH}}$ is the black hole mass. We set $\rho_{*}(r)=\rho_{\mathrm{H}}(r)$ from Equation (10) and $M_{\mathrm{BH}}=10 M_{\odot}$ or $100 M_{\odot}$.

In identifying a potential density profile IMBHs of mass $\sim 10^{3} M_{\odot}$, we consider the results of $N$-body simulations performed by Zwart et al. (2006) that predict $\sim 50$ such black holes will be present within 10 pc of Sgr A* Given that general estimations of the final mass-segregated distribution of stellar remnants range from flat-cores to extreme cusps (Alexander \& Hopman 2009; Merritt 2010, e.g), we adopt a fiducial $\rho \propto r^{-2}$ model:

$\rho_{\mathrm{Z} 06}(r)=3.98 \times 10^{2}\left(\frac{r}{\mathrm{pc}}\right)^{-2} M_{\odot} \mathrm{pc}^{-3}$

For IMBHs of mass $\sim 10^{4} M_{\odot}$, we use the density profile obtained by Mastrobuono-Battisti et al. (2014) in their work simulating twelve in-spiralling nuclear star clusters:

$\rho_{\mathrm{M} 14}(r)=6.20 \times 10^{3}\left(\frac{r}{\mathrm{pc}}\right)^{-2.32} M_{\odot} \mathrm{pc}^{-3}$.

Given that the above density profiles diverge as $r$ approaches 0 , an inner cut-off radius is defined as the semimajor axis $a_{\mathrm{c}}$ associated with a gravitational wave time for coalescence $T_{c}$ equalling Hubble time (Peters 1964),

$$
\begin{aligned}
a & \geq 4\left(\frac{1}{5} \frac{G^{3}\left(M_{\mathrm{Sgr}}+M_{\mathrm{BH}}\right) M_{\mathrm{Sgr}} M_{\mathrm{BH}}}{c^{5}} T_{\mathrm{c}}\right)^{1 / 4} \\
& =0.7 \mathrm{mpc}\left(\frac{M_{\mathrm{BH}}}{10^{3} M_{\odot}}\right)^{1 / 4} .
\end{aligned}
$$

\section{SIMULATION RESULTS}

\subsection{Measured Gravitational Effect on Sgr A*}

We calculated the relative angular deviation of Sgr A* measured at simulation times $t=1 \mathrm{yr}, 5 \mathrm{yr}, 10 \mathrm{yr}$, and $15 \mathrm{yr}$, along with the velocity magnitude and velocity component magnitude perpendicular to the Galactic plane. To compute this perpendicular component, we assume it is equal on average to the additional orthogonal velocity components, implying $v_{\perp}=v / \sqrt{3}$ where $v$ is the total $3 \mathrm{D}$ velocity amplitude. The result summaries are further supplemented by Tables 2 and 3 , which list the mean and standard deviation of the distributed values. In converting distance to subtended angle, we assume a distance from the galactic centre of $R_{0}=8.0 \mathrm{kpc}$ (Reid \& Brunthaler 2004).

For the run with background stars, the distribution of the angular shift of $\mathrm{Sgr} \mathrm{A}^{*}$ relative to its initial position after a time period of 15 years was best fit by a gamma distribution with mean $22.7 \pm 5.0 \mu$ as. The distribution of velocity magnitudes for $\mathrm{Sgr} \mathrm{A}^{*}$ due to the presence of this profile was fit by a Maxwell-Boltzmann distribution with mean $0.06 \pm 0.02 \mathrm{~km} \mathrm{~s}^{-1}$, and a perpendicular component magnitude of $0.04 \pm 0.01 \mathrm{~km} \mathrm{~s}^{-1}$.
An average of $1146210 M_{\odot}$ black holes and $358100 M_{\odot}$ black holes were drawn within the inner parsec, displacing Sgr $A^{*}$ on average by $20.6 \pm 8.5 \mu$ as and $35.5 \pm 14.9 \mu$ as respectively after 15 years. The velocity magnitude of Sgr $\mathrm{A}^{*}$ induced by the $10 M_{\odot}$ black holes was on average $0.05 \pm 0.02 \mathrm{~km} \mathrm{~s}^{-1}$, with a perpendicular component of $0.03 \pm 0.01 \mathrm{~km} \mathrm{~s}^{-1}$. For the $100 M_{\odot}$ black hole density profile, Sgr $A^{*}$ possessed a mean velocity magnitude of $0.09 \pm 0.04 \mathrm{~km} \mathrm{~s}^{-1}$, and a perpendicular component magnitude of $0.05 \pm 0.02 \mathrm{~km} \mathrm{~s}^{-1}$.

There were on average 4 IMBHs of mass $10^{3} M_{\odot}$ drawn according to our fiducial density profile within one parsec, inducing angular shifts of $39.6 \pm 25.7 \mu$ as on the position of Sgr $\mathrm{A}^{*}$ after 15 years. The average velocity of the central black hole induced by this profile was $0.1 \pm 0.06 \mathrm{~km} \mathrm{~s}^{-1}$, though unlike with other density profiles the distribution of this velocity was better fit to a general gamma distribution with mean and standard deviation $0.06 \pm 0.04 \mathrm{~km} \mathrm{~s}^{-1}$. The mean perpendicular velocity component was $0.06 \pm 0.04 \mathrm{~km} \mathrm{~s}^{-1}$.

From the Mastrobuono-Battisti et al. density profile, an average of ten $10^{4} M_{\odot}$ IMBHs were drawn in the inner parsec. After 15 years, they induced an angular shift in Sgr $\mathrm{A}^{*}$ position of $953.9 \pm 459.0 \mu \mathrm{as}$, with velocity magnitude and perpendicular component of $2.63 \pm 1.33 \mathrm{~km} \mathrm{~s}^{-1}$ and $1.57 \pm$ $0.86 \mathrm{~km} \mathrm{~s}^{-1}$ respectively.

\subsection{Effects on Orbital Parameters of S2}

Table 4 provides a thorough summary of the average changes in semi-major axis $(\Delta a / a$, where $a=4.823 \mathrm{mpc})$, eccentricity $(\Delta e)$, inclination $(\Delta i)$, longitude of ascending node $(\Delta \Omega)$, shift of periapse $\left(\Delta \Theta_{p}\right)$, and shift of apoapse $\left(\Delta \Theta_{a}\right)$ induced by the stellar control and the tested discrete and smooth stellar/IMBH density profiles. For all elements, the mean change did not scale simply with mass; $10^{4} M_{\odot}$ IMBHs produced the maximal average change in an orbital element, with the stellar control and $10 M_{\odot}$ stellar black holes producing the next largest average changes, and the $10^{3} M_{\odot}$ IMBHs followed by the $100 M_{\odot}$ stellar black holes producing the least. Discretely realized profiles induced various mean changes in semi-major axis, differing by approximately a factor of ten $\left(\Delta a / a \sim 10^{-5}-10^{-4}\right)$ and possessing no dramatic differences from changes in semi-major axis induced by a smoothly distributed profile. Stars and low-mass stellar black holes induced changes on eccentricity with a mean of $\sim 10^{-5}$, while for $100 M_{\odot}$ and $10^{3} M_{\odot}$ black holes this value was $\sim 6 \times 10^{-6}$. The mean change in eccentricity due to $10^{4} M_{\odot}$ black holes was almost a factor of 100 greater at $3.76 \times 10^{-4}$. The changes in eccentricity induced by discretely distributed particles was less in all cases than those induced by the corresponding smooth simulated profile, though by zero to two orders of magnitude.

The second row panels of Figure 16 show that smooth profiles had absolutely negligible effects on inclination and longitude of ascending node (all changing these elements by $\sim 10^{-13}$ degrees) in contrast to their discrete counterparts. Discretely distributed $10^{4} M_{\odot}$ IMBHs induced the largest average change of $\Delta i=0.0539^{\circ}$ and $\Delta \Omega=0.152^{\circ} .1000 M_{\odot}$ and stellar black holes of masses $10 M_{\odot}$ and $100 M_{\odot}$ induced average changes of $\Delta i \approx 0.001^{\circ}$ and $\Delta \Omega \approx 0.003^{\circ}$, and the stellar control induced changes in $i$ and $\Omega$ of $\sim 0.003^{\circ}$ and $\sim 0.016^{\circ}$ respectively. 
Angular displacement of Sgr A* ( $\mu$ as)

\begin{tabular}{|c|c|c|c|c|c|c|c|c|c|c|}
\hline & \multicolumn{2}{|c|}{$\left\langle m_{\rho}\right\rangle=1 M_{\odot}$} & \multicolumn{2}{|c|}{$\left\langle m_{\rho}\right\rangle=10 M_{\odot}$} & \multicolumn{2}{|c|}{$\left\langle m_{\rho}\right\rangle=100 M_{\odot}$} & \multicolumn{2}{|c|}{$\left\langle m_{\rho}\right\rangle=10^{3} M_{\odot}$} & \multicolumn{2}{|c|}{$\left\langle m_{\rho}\right\rangle=10^{4} M_{\odot}$} \\
\hline & $\mu$ & $\sigma$ & $\mu$ & $\sigma$ & $\mu$ & $\sigma$ & $\mu$ & $\sigma$ & $\mu$ & $\sigma$ \\
\hline $1 \mathrm{yr}$ & 0.7 & 0.3 & 1.2 & 0.6 & 2.4 & 1 & 2.8 & 1.9 & 67.8 & 33.8 \\
\hline $5 \mathrm{yr}$ & 5.8 & 1.66 & 6.9 & 2.8 & 11.9 & 5 & 13.6 & 9.2 & 333.8 & 165.8 \\
\hline $10 \mathrm{yr}$ & 14.4 & 3.4 & 12.7 & 5.6 & 23.6 & 10 & 26.8 & 17.8 & 654.9 & 321.2 \\
\hline $15 \mathrm{yr}$ & 22.7 & 5 & 20.6 & 8.5 & 35.5 & 14.9 & 39.6 & 25.7 & 953.9 & 459 \\
\hline
\end{tabular}

Table 2. Mean $(\mu)$ and standard deviation $(\sigma)$ of the angular displacement of Sgr A*, measured relative to its original position, induced by discrete density profiles describing the distribution of stars $\left(\left\langle m_{\rho}\right\rangle=1 M_{\odot}\right)$, stellar black holes $\left(\left\langle m_{\rho}\right\rangle=10 M_{\odot}, 100 M_{\odot}\right)$, and intermediate mass black holes $\left(\left\langle m_{\rho}\right\rangle=1000 M_{\odot}, 10^{4} M_{\odot}\right)$. See Figures 1, 4, 7, 10, and 13 for its visual representation.

\begin{tabular}{|c|c|c|c|c|c|c|c|c|c|c|}
\hline & \multicolumn{2}{|c|}{$\left\langle m_{\rho}\right\rangle=1 M_{\odot}$} & \multicolumn{2}{|c|}{$\left\langle m_{\rho}\right\rangle=10 M_{\odot}$} & \multicolumn{2}{|c|}{$\left\langle m_{\rho}\right\rangle=100 M_{\odot}$} & \multicolumn{2}{|c|}{$\left\langle m_{\rho}\right\rangle=10^{3} M_{\odot}$} & \multicolumn{2}{|c|}{$\left\langle m_{\rho}\right\rangle=10^{4} M_{\odot}$} \\
\hline & $\mu$ & $\sigma$ & $\mu$ & $\sigma$ & $\mu$ & $\sigma$ & $\mu$ & $\sigma$ & $\mu$ & $\sigma$ \\
\hline$|v|\left(\mathrm{km} \mathrm{s}^{-1}\right)$ & 0.06 & 0.02 & 0.05 & 0.02 & 0.09 & 0.04 & 0.1 & 0.06 & 2.63 & 1.33 \\
\hline$\left|v_{\perp}\right|\left(\mathrm{km} \mathrm{s}^{-1}\right)$ & 0.04 & 0.01 & 0.03 & 0.01 & 0.05 & 0.02 & 0.06 & 0.04 & 1.57 & 0.86 \\
\hline
\end{tabular}

Table 3. Mean $(\boldsymbol{\mu})$ and standard deviation $(\sigma)$ of the absolute intrinsic velocity and its perpendicular component of Sgr A*, induced by discrete density profiles describing the distribution of stars $\left(\left\langle m_{\rho}\right\rangle=1 M_{\odot}\right)$, stellar black holes $\left(\left\langle m_{\rho}\right\rangle=10 M_{\odot}, 100 M_{\odot}\right)$, and intermediate mass black holes $\left(\left\langle m_{\rho}\right\rangle=1000 M_{\odot}, 10^{4} M_{\odot}\right)$. The table is visualized in Figures 3, 6, 9, 12, and 15 .
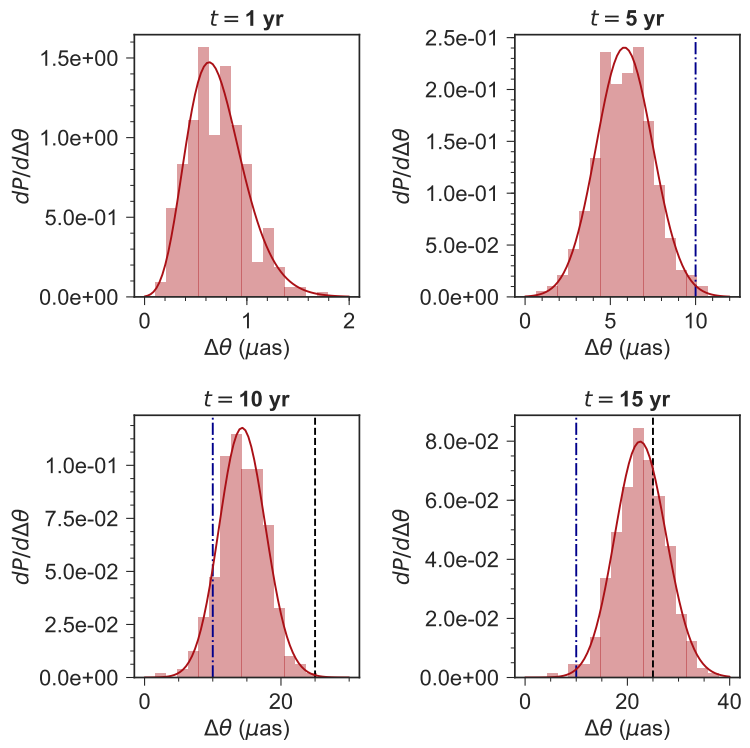

Figure 1: Probability distribution of angular displacement $(\Delta \theta)$ of $\operatorname{Sgr~} \mathrm{A}^{*}$ at four times $(\Delta t=1 \mathrm{yr}, 5 \mathrm{yr}, 10 \mathrm{yr}$, and $15 \mathrm{yr})$, when surrounded by stellar control. Each histogram is normalized to unity and fitted to a gamma distribution (marked by the solid curve) with mean and standard deviation recorded in Table 2. Blue dot-dashed lines and black dashed in the histogram of angular displacement indicate the potential angular precision of GRAVITY (10 $\mu$ as) and the EHT (25 $\mu$ as) respectively.

Discretely distributed profiles also generated larger shifts in periapse and apoapse when compared to smooth distributions. For all but the $10^{4} M_{\odot}$ IMBH profile the mean angular shift of periapse position was $\Delta \Theta_{p} \lesssim 8 \mu$ as. Shifts in apoapse position $\Delta \Theta_{a}$ were greater for each profile than $\Delta \Theta_{p}$, reaching a maximum mean of 0.97 mas with the $10^{4} M_{\odot}$ IMBHs. All profiles induced a mean $\Delta \Theta_{a}$ that was above a
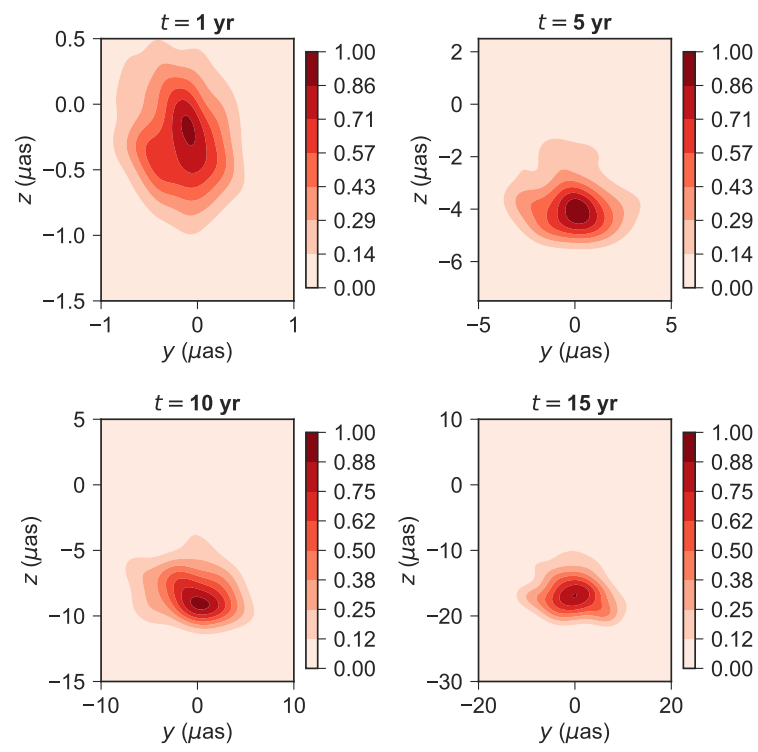

Figure 2: Bivariate probability density of Sgr $A^{*}$ at four times $(\Delta t=1 \mathrm{yr}, 5 \mathrm{yr}, 10 \mathrm{yr}$, and $15 \mathrm{yr})$, when surrounded by stellar control.

$10 \mu$ as threshold, reaching a next-largest value of $130 \mu$ as with the stellar control, $27.6 \mu$ as with $10 M_{\odot}$ black holes, $19.8 \mu$ as with $1000 M_{\odot}$ black holes, and $13.7 \mu$ as with $100 M_{\odot}$ black holes.

\section{DISCUSSION}

Though the simulations carried out in this work were based on simplified assumptions, their results provide non-trivial insight into which black hole populations may be detectable in the Galactic Centre. Below we compare obtained results to our strongest current observational constraints, which 

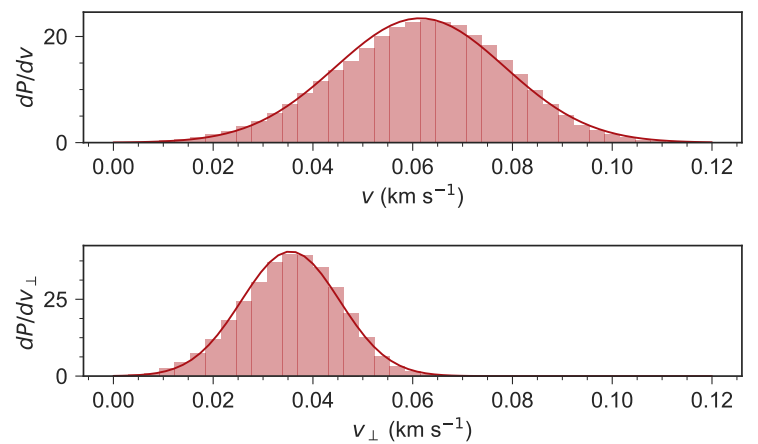

Figure 3: Probability distribution of velocity of Sgr A* when surrounded by stellar control. The upper panel displays the distribution of total velocity magnitude $(v)$ measured at each time step of a simulation, while the lower panel shows the distribution of measured velocity component perpendicular to the galactic disk $\left(v_{\perp}\right)$. Solid lines indicate fitted distributions with means and standards deviation noted in Table 3. Total velocity magnitude is fitted to a MaxwellBoltzmann distribution while perpendicular velocity magnitude is fitted to a gamma distribution, and the area under each curve is normalized to unity.
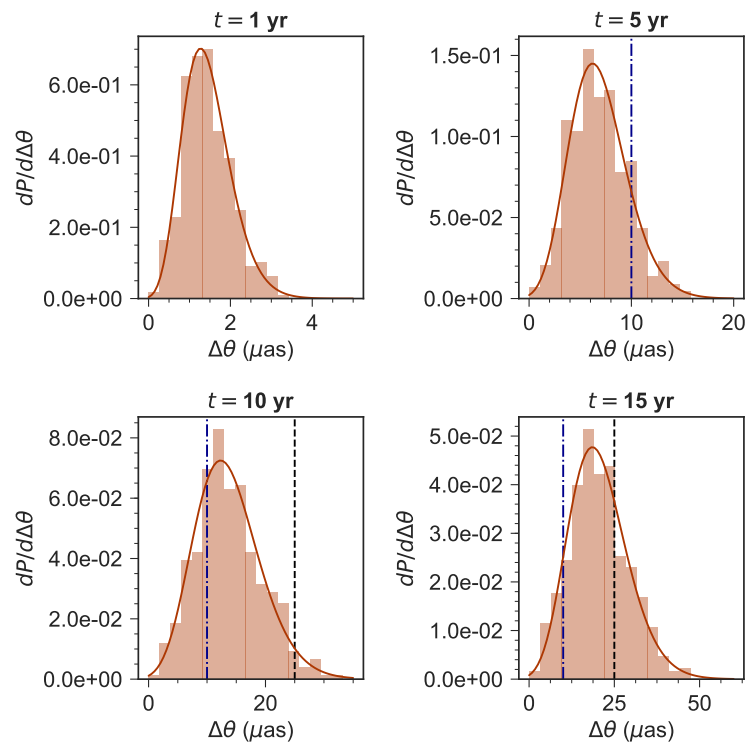

Figure 4: As in Figure 1, with Sgr A* surrounded by a population of $10 M_{\odot}$ stellar black holes.

rely on the proper motion of Sgr A* possessing a $2 \sigma$ upper limit of $1.8 \mathrm{~km} \mathrm{~s}^{-1}$ for the velocity perpendicular to the galactic plane (Reid \& Brunthaler 2004), a $\sigma$ upper limit of $1.3 \mathrm{~km} \mathrm{~s}^{-1}$ in the direction of galactic rotation (Reid et al. 2009), and the constrained error of Gillessen et al. (2009) orbital fit for S2.

With the constraint of proper motion in mind, all stellar mass and intermediate mass black hole profiles under consideration are allowed. The combined precision of the EHT in sub-array mode ( $225 \mu$ as, see Broderick et al. 2011) would allow detection within 15 years of the angular dis-
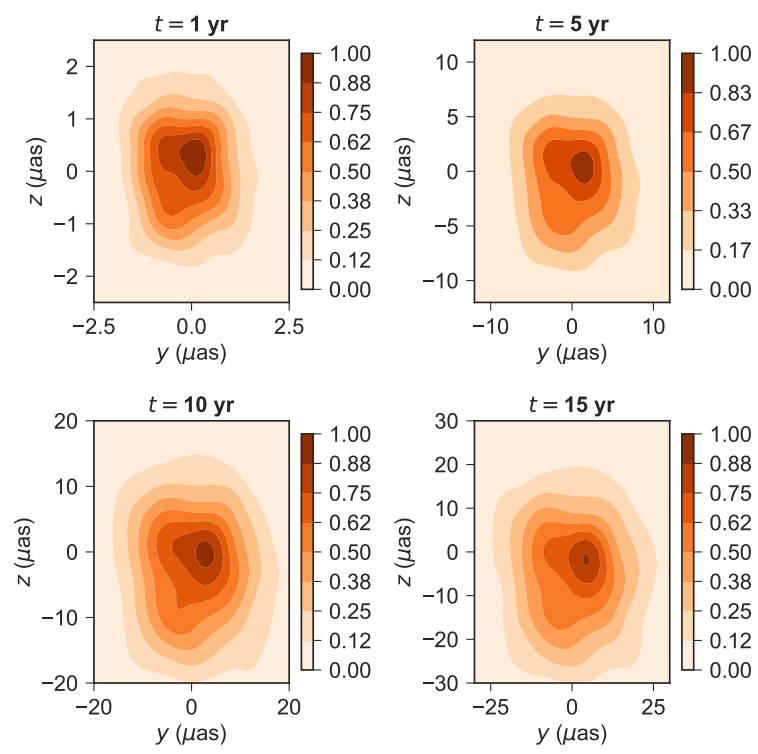

Figure 5: As in Figure 2, with $\mathrm{Sgr} \mathrm{A}^{*}$ surrounded by a population of $10 M_{\odot}$ stellar black holes.
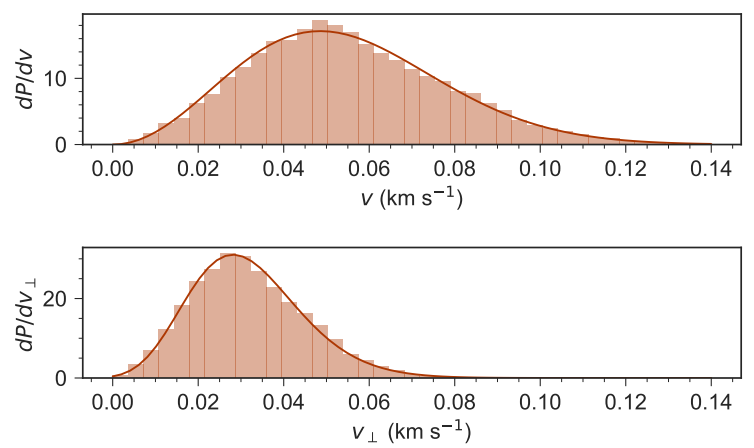

Figure 6: As in Figure 3, with Sgr A* surrounded by a population of $10 M_{\odot}$ stellar black holes.

placement induced on Sgr A* by any of the examined profiles. The velocity kick given to on Sgr A* by stars and stellar black holes $\left(\sim 0.06 \mathrm{~km} \mathrm{~s}^{-1}\right)$ stand in agreement with an amplitude of Brownian motion calculated by Merritt et al. (2007); Chatterjee et al. (2002); Loeb \& Furlanetto (2013), using $\left\langle m_{*}\right\rangle=1 M_{\odot}$ and $M_{\mathrm{BH}}=4.4 \times 10^{6} M_{\odot}$ (See Equation (1.18)), as well as $N$-body simulations of stars within the inner 2 pc performed by Reid \& Brunthaler (2004). Both $10^{2} M_{\odot}$ and $10^{3} M_{\odot}$ black holes induce angular shifts in the position of $\mathrm{Sgr} \mathrm{A}^{*}$ at a rate of $\gtrsim 2 \mu$ as $\mathrm{yr}^{-1}$. The mean velocity of Sgr A* when surrounded by each profile is on the order of $0.1 \mathrm{~km} \mathrm{~s}^{-1}$, closer to estimates on velocity out of the disk as outlined by Reid \& Brunthaler (2004) than results from other profiles. However, due to their similar dynamical signature the tested profiles for $10^{2} M_{\odot}$ and $100^{2} M_{\odot}$ black holes are arguably impossible to differentiate from their gravitational effect on Sgr A* alone. The $10^{4} M_{\odot} \mathrm{IMBH}$ profile produced the largest angular shifts, with a mean of $\sim 65 \mu \mathrm{as} \mathrm{yr}^{-1}$. It additionally induced the largest intrinsic velocity of $2.62 \pm 1.35 \mathrm{~km} \mathrm{~s}^{-1}$. The perpendicular component 

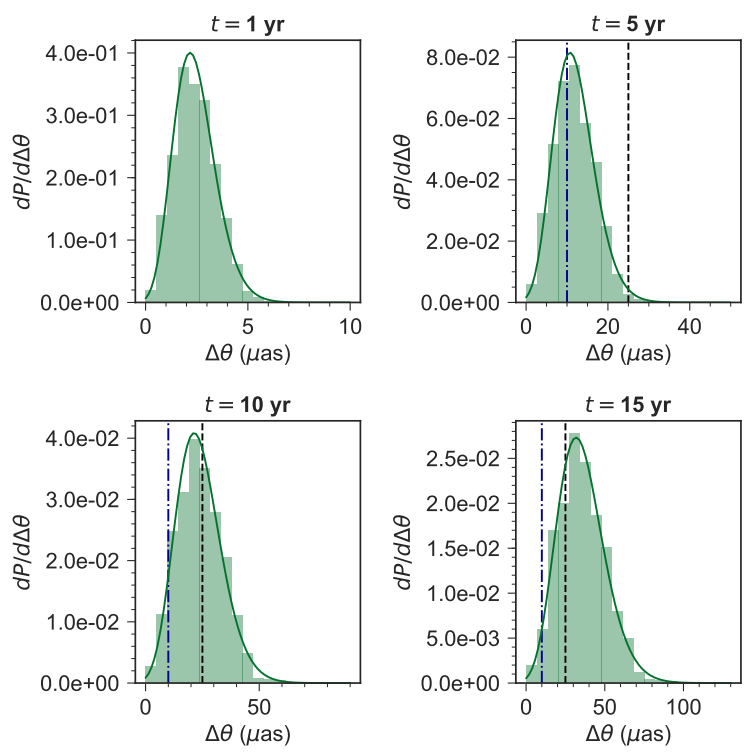

Figure 7: As in Figure 1, with Sgr A* surrounded by a population of $100 M_{\odot}$ stellar black holes.
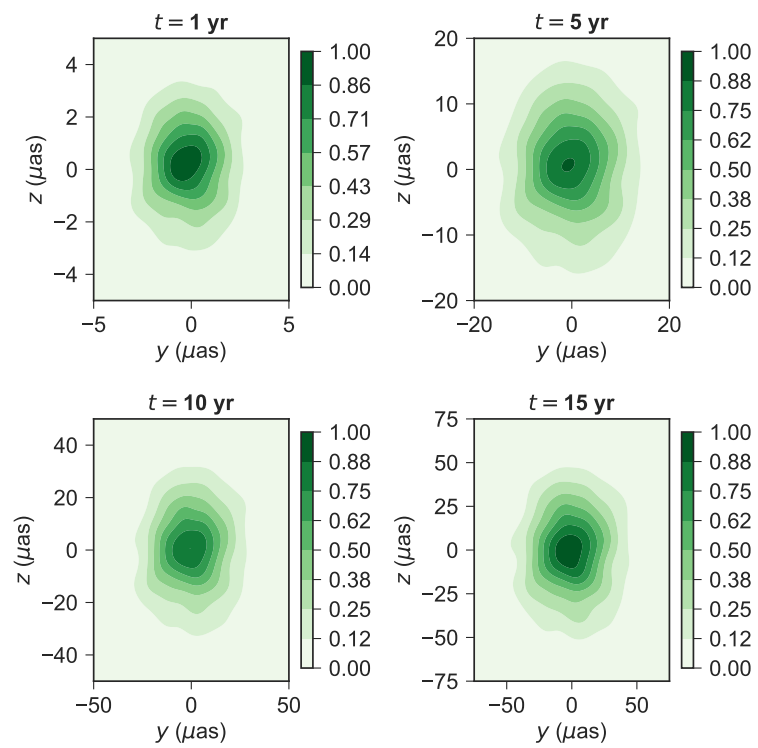

Figure 8: As in Figure 2, with $\mathrm{Sgr} \mathrm{A}^{*}$ surrounded by a population of $100 M_{\odot}$ stellar black holes.

of this was $1.6 \pm 0.9 \mathrm{~km} \mathrm{~s}^{-1}$, the mean of which is just within the present $2 \sigma$ limit of $1.8 \mathrm{~km} \mathrm{~s}^{-1}$ (Reid \& Brunthaler 2004).

To properly examine the impact of these density profiles on S2, we first review the current accuracies in VLT and Keck data embedded in errors allotted for by Gillessen et al. (2009). The best fitted values for semi-major axis and eccentricity are accurate to $\sim 2$ mas and $3 \times 10^{-3}$ respectively, and the inclination, longitude of ascending note, and argument of periapse are accurate to $0.72^{\circ}-0.81^{\circ}$. To properly use the orbit of $\mathrm{S} 2$ as a probe for stellar remnants and IMBHs, the changes induced by these dark objects must necessarily fit within these accuracies while additionally surpassing
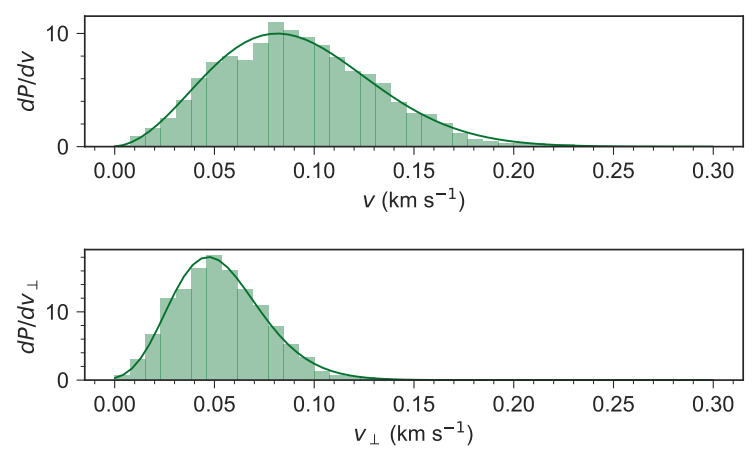

Figure 9: As in Figure 3, with Sgr A* surrounded by a population of $100 M_{\odot}$ stellar black holes.
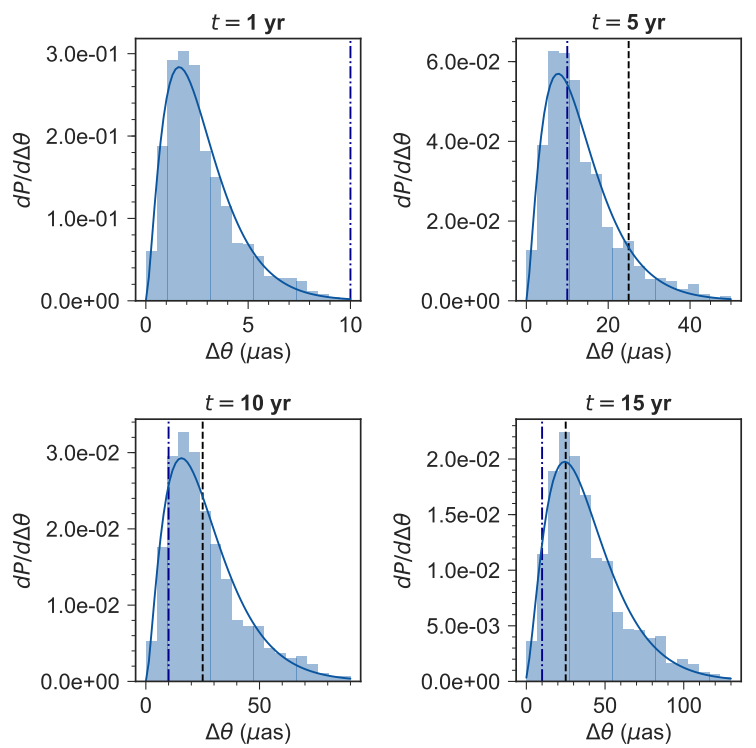

Figure 10: As in Figure 1, with Sgr $A^{*}$ surrounded by a population of $10^{3} M_{\odot}$ IMBHs.

orbital changes associated with relativistic effects. Grould et al. (2017) have investigated the potential of GRAVITY to detect various relativistic effects on the S2, concluding that a shift of periapse due to relativistic advance will be $30 \mu$ as in 14 years (roughly $34 \mu$ as per revolution). Alternatively Gualandris et al. (2010) calculated the displacement in the star's apoapse as

$\Delta r_{a} \approx a(1+e) \Delta \varpi \approx \frac{6 \pi G M_{\mathrm{Sgr}}}{c^{2}(1-e)}$,

where $\Delta \varpi=\Delta(\Omega+\omega)$ is the advance in periapse angle. Using $M_{\text {Sgr }}=4.4 \times 10^{6} M_{\odot}$ and $R_{0}=8 \mathrm{kpc}, \Delta r_{a}$ subtends an angle on the sky, $\Delta \Theta_{a}=0.86$ mas. The angle on the sky subtended by the displacement in the star's periapse can then be calculated as $\Delta \Theta_{p}=\Delta \Theta_{a}(1-e) /(1+e) \approx 54.9 \mu$ as. Thus in $\Delta \Theta_{a}$ and $\Delta \Theta_{p}$, along with constrained errors of $a, e, i, \Omega$, and $\omega$, we have lower limits and upper limits respectively to determine the detectability of a certain density profile's dynamical signature on S2.

There is a notable difference between the dynamical ef- 

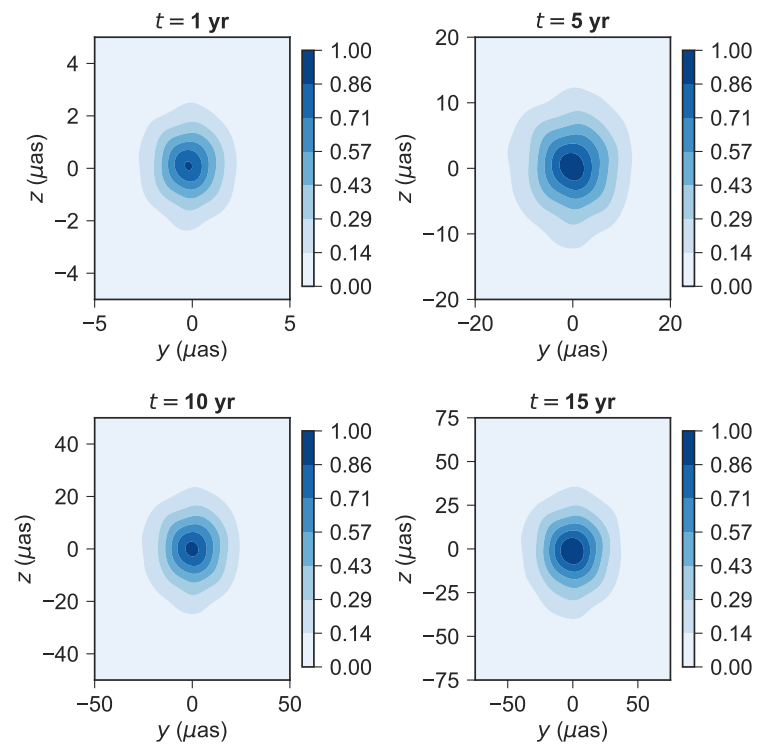

Figure 11: As in Figure 2, with Sgr A* surrounded by a population of $10^{3} M_{\odot}$ IMBHs.
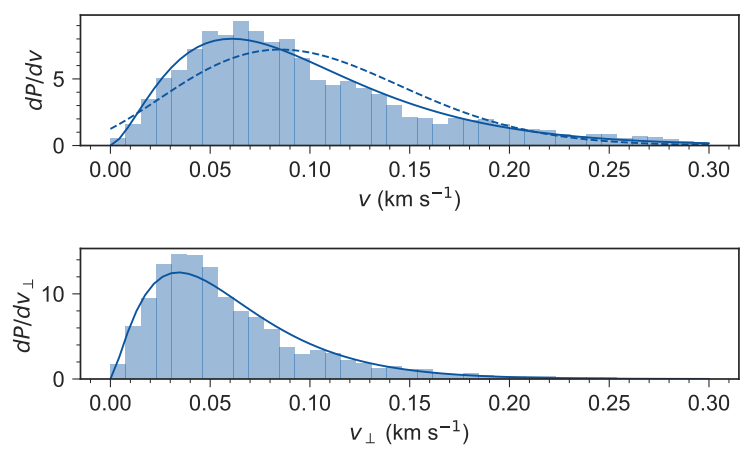

Figure 12: As in Figure 3, with $\mathrm{Sgr} \mathrm{A}^{*}$ surrounded by a population of $10^{3} M_{\odot}$ IMBHs. Unlike Figure 3, the solid line in the upper panel outlines a fitted gamma distribution while the dashed line marks a fitted Maxwellian distribution.

fect of smooth and discrete distributions in orbital arguments pertaining to angle ( $i$ and $\Omega$ ). A smooth or centralized distribution creates virtually no changes in these parameters, whereas the gravitational effect of discretely distributed particles generates distinct apsidal precession of the orbit of S2. The average change in $i$ and $\Omega$ for all density profiles are within current error of $0.7^{\circ}$. It is interesting to note that the mean change in inclination and longitude of ascending node due to the stellar control and $10 M_{\odot}$ stellar mass black holes exceeds that induced by $10^{2} M_{\odot}$ stellar black holes and $10^{3} M_{\odot}$ IMBHs. This may be because, due to their increased number density, these smaller objects experience closer encounters with S2 that more intensely perturb its orbit. However, stars, stellar black holes, and $10^{3} M_{\odot}$ IMBHs all induced changes in periapse and apoapse under estimated relativistic shifts, implying that these density profiles are not detectable through observation of S2's orbital precession. Only the $10^{4} M_{\odot} \mathrm{IMBH}$ profile induced changes
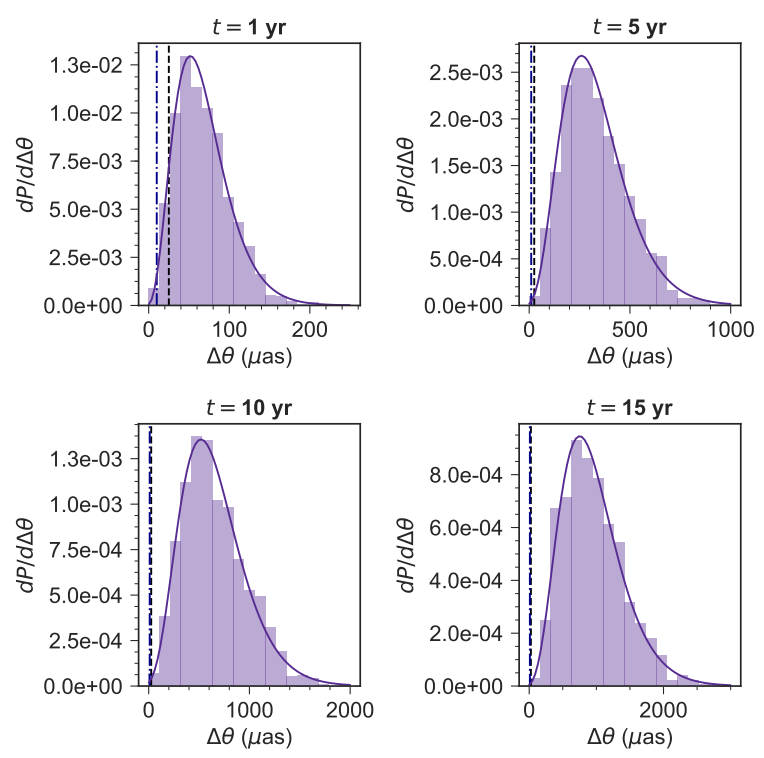

Figure 13: As in Figure 1, with Sgr A* surrounded by a population of $10^{4} M_{\odot}$ IMBHs.
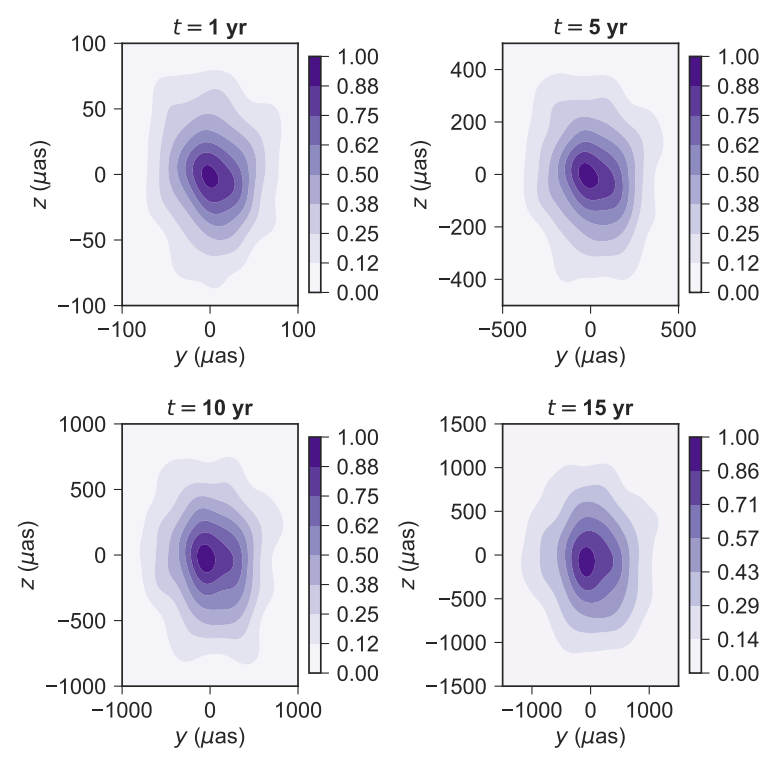

Figure 14: As in Figure 2, with Sgr A* surrounded by a population of $10^{4} M_{\odot}$ IMBHs.

in these parameters exceeding the lower limit placed by the relativistic effects. With this, S2 appears to be a more effective probe for detecting IMBHs of mass $\gtrsim 10^{4} M_{\odot}$. Particularly through observations of $\sim$ milli-arcsecond angular shifts in apoapse, along with perceptible changes in inclination and longitude of ascending node, we can infer the existence of surrounding discrete objects.

This work can be expanded upon by an inclusion of a larger variety of density profiles and improved numerical methods. An asymmetric distribution of particles would generate a larger amplitude wobble; this may have already played a role in simulations of the $10^{4} M_{\odot}$ IMBH profile, 

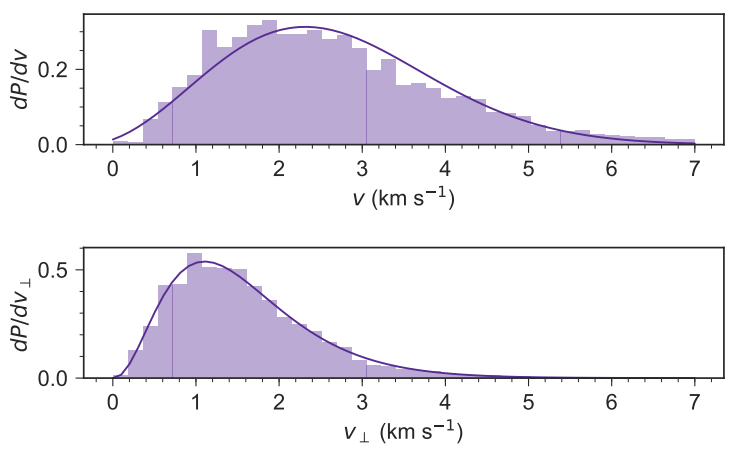

Figure 15: As in Figure 3, with $\mathrm{Sgr} \mathrm{A}^{*}$ surrounded by a population of $10^{4} M_{\odot}$ IMBHs.

where the assumed spherical symmetry was compromised by a relatively small number density and larger gravitational effects on Sgr A* and S2 were generated. Even though this work identified a potentially observable IMBH black hole density profile through its gravitational effects on S2, this effect may be replicable by alternative unseen matter or a single large IMBH. Thus, it is critical to understand how the magnitude of these changes evolve over multiple periods of S2's orbit.

\section{ACKNOWLEDGEMENTS}

This work was supported in part by the Mellon Mays Foundation and by the Black Hole Initiative at Harvard University, which is funded by a grant from the John Templeton Foundation.

\section{REFERENCES}

Abel T., Bryan G. L., Norman M. L., 2000, The Astrophysical Journal, 540, 39

Alexander T., Hopman C., 2009, The Astrophysical Journal, 697, 1861

Alig C., Burkert A., Johansson P. H., Schartmann M., 2011, Monthly Notices of the Royal Astronomical Society, 412, 469

Alig C., Schartmann M., Burkert A., Dolag K., 2013, The Astrophysical Journal, 771, 119

Antonini F., Capuzzo-Dolcetta R., Mastrobuono-Battisti A., Merritt D., 2012, The Astrophysical Journal, 750, 111

Binney J., Tremaine S., 2011, Galactic dynamics. Princeton university press

Bonnell I., Rice W., 2008, Science, 321, 1060

Broderick A. E., Loeb A., Reid M. J., 2011, The Astrophysical Journal, 735, 57

Bromley B. C., Kenyon S. J., Geller M. J., Barcikowski E., Brown W. R., Kurtz M. J., 2006, The Astrophysical Journal, 653, 1194

Bromm V., Coppi P. S., Larson R. B., 1999, The Astrophysical Journal, 527, L5

Chandrasekhar S., 1941, The Astrophysical Journal, 94, 511

Chandrasekhar S., 1943, The Astrophysical Journal, 97, 255

Chatterjee P., Hernquist L., Loeb A., 2002, The Astrophysical Journal, 572,371

Ebisuzaki T., et al., 2001, The Astrophysical Journal Letters, 562, L19
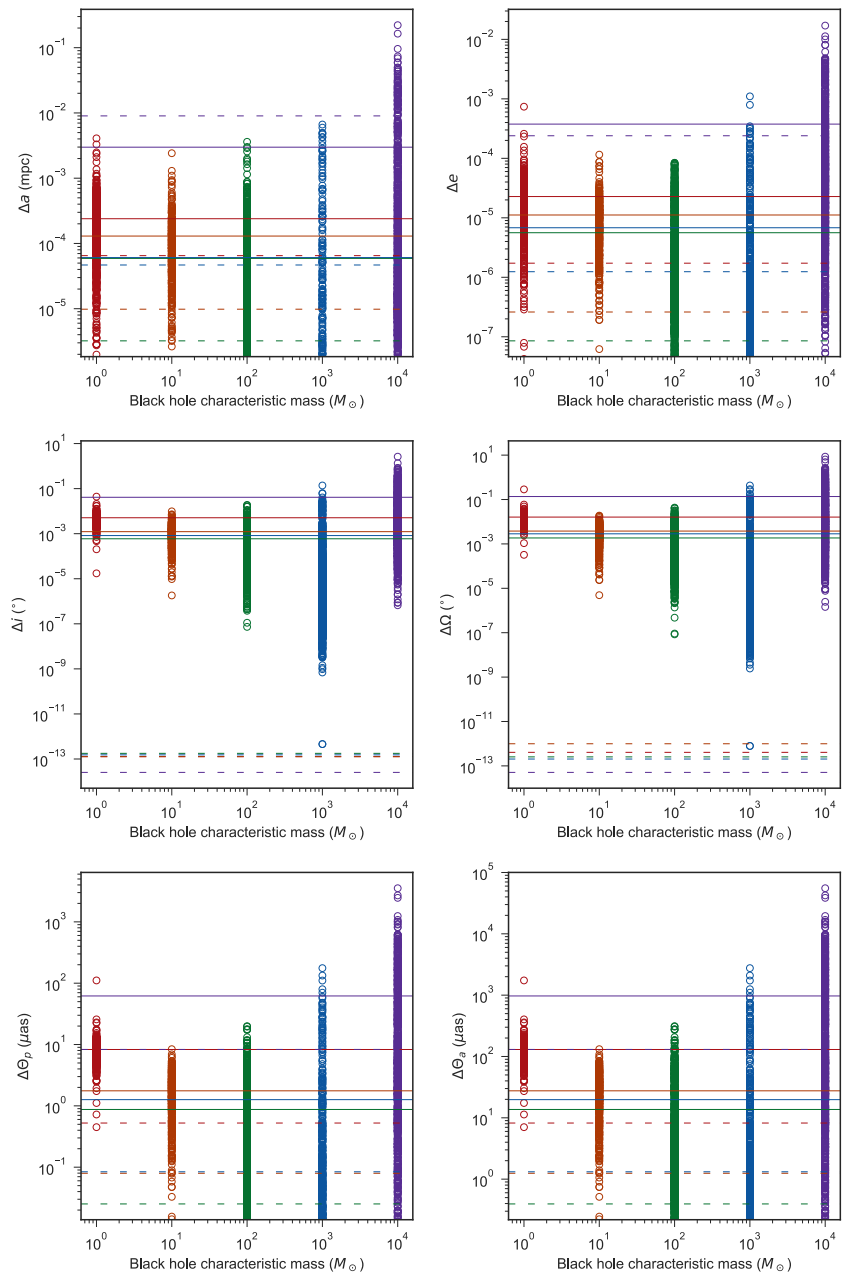

Figure 16: Changes in orbital elements (semi-major axis, eccentricity, inclination, longitude of ascending node, shift of periapse, and shift of apoapse) experienced by S2 during each simulation run when surrounded by tested density profiles. Solid lines indicate the average change of the orbital element induced by a certain density profile, with characteristic mass indicated on the $\mathrm{x}$-axis and coordinated by colour. Dashed lines indicate the average change of the orbital element induced by a smooth version of a certain density profile, also marked by colour. A single circle indicates the change in orbital element that occurred in one simulation of S2 embedded in a particular density profile.

Figer D. F., Najarro F., Morris M., McLean I. S., Geballe T. R., Ghez A. M., Langer N., 1996, The Astrophysical Journal, 506, 384

Freitag M., Amaro-Seoane P., Kalogera V., 2006, The Astrophysical Journal, 649, 91

Fujii M., Iwasawa M., Funato Y., Makino J., 2009, The Astrophysical Journal, 695, 1421

Fujii M., Iwasawa M., Funato Y., Makino J., 2010, The Astrophysical Journal Letters, 716, L80

Ghez A., et al., 2003, The Astrophysical Journal Letters, 586, L127

Ghez A., et al., 2008, The Astrophysical Journal, 689, 1044

Giersz M., Leigh N., Hypki A., Askar A., Lützgendorf N., 2016, Formation mechanisms of IMBH in globular clusters, arXiv preprint arXiv:1607.08384 


\begin{tabular}{|c|c|c|c|c|c|c|}
\hline Orbital element & Mean change & $\left\langle m_{\rho}\right\rangle=1 M_{\odot}$ & $\left\langle m_{\rho}\right\rangle=10 M_{\odot}$ & $\left\langle m_{\rho}\right\rangle=100 M_{\odot}$ & $\left\langle m_{\rho}\right\rangle=10^{3} M_{\odot}$ & $\left\langle m_{\rho}\right\rangle=10^{4} M_{\odot}$ \\
\hline \multirow{2}{*}{$\Delta a / a$} & Discrete & 4.14E-05 & $2.07 \mathrm{E}-05$ & $1.22 \mathrm{E}-05$ & $1.26 \mathrm{E}-05$ & $6.21 \mathrm{E}-04$ \\
\hline & Smooth & $1.35 \mathrm{E}-05$ & $2.03 \mathrm{E}-06$ & $6.63 \mathrm{E}-07$ & $9.67 \mathrm{E}-06$ & $1.86 \mathrm{E}-03$ \\
\hline \multirow{2}{*}{$\Delta e$} & Discrete & $2.28 \mathrm{E}-05$ & $1.12 \mathrm{E}-05$ & $5.61 \mathrm{E}-06$ & $6.81 \mathrm{E}-06$ & $3.76 \mathrm{E}-04$ \\
\hline & Smooth & $1.73 \mathrm{E}-06$ & $2.61 \mathrm{E}-07$ & $8.53 \mathrm{E}-08$ & $1.24 \mathrm{E}-06$ & $2.40 \mathrm{E}-04$ \\
\hline \multirow{2}{*}{$\Delta i(\operatorname{deg})$} & Discrete & $5.10 \mathrm{E}-03$ & $1.23 \mathrm{E}-03$ & $5.94 \mathrm{E}-04$ & $8.25 \mathrm{E}-04$ & 4.13E-02 \\
\hline & Smooth & $1.27 \mathrm{E}-13$ & $1.27 \mathrm{E}-13$ & $1.78 \mathrm{E}-13$ & $1.53 \mathrm{E}-13$ & $2.54 \mathrm{E}-14$ \\
\hline \multirow{2}{*}{$\Delta \Omega(\operatorname{deg})$} & Discrete & 1.62E-02 & 3.79E-03 & $1.86 \mathrm{E}-03$ & $2.85 \mathrm{E}-03$ & $1.36 \mathrm{E}-01$ \\
\hline & Smooth & $4.07 \mathrm{E}-13$ & $9.92 \mathrm{E}-13$ & $2.54 \mathrm{E}-13$ & $2.04 \mathrm{E}-13$ & $5.09 \mathrm{E}-14$ \\
\hline \multirow{2}{*}{$\Delta \Theta_{p}(\mu \mathrm{as})$} & Discrete & 8.3 & 1.8 & 0.9 & 1.3 & 61.9 \\
\hline & Smooth & 0.5 & 0.08 & 0.03 & 0.08 & 8.3 \\
\hline \multirow{2}{*}{$\Delta \Theta_{a}(\mu \mathrm{as})$} & Discrete & 130 & 27.6 & 13.7 & 19.8 & 970.4 \\
\hline & Smooth & 8.2 & 1.3 & 0.4 & 1.3 & 130.2 \\
\hline
\end{tabular}

Table 4. Average changes induced by discrete and smooth density profiles describing the distribution of stars $\left(\left\langle m_{\rho}\right\rangle=1 M_{\odot}\right)$, stellar black holes $\left(\left\langle m_{\rho}\right\rangle=10 M_{\odot}, 100 M_{\odot}\right)$, and intermediate mass black holes $\left(\left\langle m_{\rho}\right\rangle=1000 M_{\odot}, 10^{4} M_{\odot}\right)$ on the the semi-major axis $(\Delta a / a$, with $a \sim 5 \mathrm{mpc})$, eccentricity $(e)$, inclination $(i)$, longitude of the periapsis $(\Omega)$, angular shift of periapse $\left(\Delta \Theta_{p}\right)$, and angular shift of apoapse $\left(\Delta \Theta_{a}\right)$ of $\mathrm{S} 2$.

Gillessen S., Eisenhauer F., Trippe S., Alexander T., Genzel R., Martins F., Ott T., 2009, The Astrophysical Journal, 692, 1075

Grould M., Vincent F., Paumard T., Perrin G., 2017, Astronomy \& Astrophysics, 608, A60

Gualandris A., Merritt D., 2009, The Astrophysical Journal, 705, 361

Gualandris A., Gillessen S., Merritt D., 2010, Monthly Notices of the Royal Astronomical Society, 409, 1146

Gürkan M. A., Freitag M., Rasio F. A., 2004, The Astrophysical Journal, 604, 632

Hansen B. M., Milosavljević M., 2003, The Astrophysical Journal Letters, 593, L77

Hernquist L., 1990, The Astrophysical Journal, 356, 359

Leigh N. W., Böker T., Maccarone T. J., Perets H. B., 2013, Monthly Notices of the Royal Astronomical Society, 429, 2997

Loeb A., Furlanetto S. R., 2013, The first galaxies in the universe. Princeton University Press

Lu J., Ghez A., Hornstein S., Morris M., Becklin E., Matthews K., 2008, The Astrophysical Journal, 690, 1463

Mapelli M., Hayfield T., Mayer L., Wadsley J., 2008, arXiv preprint arXiv:0805.0185

Mastrobuono-Battisti A., Perets H. B., Loeb A., 2014, The Astrophysical Journal, 796, 40

Merritt D., 2010, The Astrophysical Journal, 718, 739

Merritt D., Berczik P., Laun F., 2007, The Astronomical Journal, 133,553

Merritt D., Gualandris A., Mikkola S., 2009, The Astrophysical Journal Letters, 693, L35

Miller M. C., Freitag M., Hamilton D. P., Lauburg V. M., 2005, The Astrophysical Journal Letters, 631, L117

Miralda-Escudé J., Gould A., 2000, The Astrophysical Journal, 545,847

Morris M., 1993, The Astrophysical Journal, 408, 496

Paumard T., et al., 2006, The Astrophysical Journal, 643, 1011

Perets H. B., Hopman C., Alexander T., 2007, The Astrophysical Journal, 656, 709

Perets H. B., Gualandris A., Kupi G., Merritt D., Alexander T., 2009, The Astrophysical Journal, 702, 884

Peters P. C., 1964, Physical Review, 136, B1124

Petts J., Gualandris A., 2017, Monthly Notices of the Royal Astronomical Society, 467, 3775

Reid M. J., Brunthaler A., 2004, The Astrophysical Journal, 616, 872

Reid M., et al., 2009, The Astrophysical Journal, 700, 137
Rein H., Liu S.-F., 2012, Astronomy \& Astrophysics, 537, A128

Rein H., Spiegel D. S., 2014, Monthly Notices of the Royal Astronomical Society, 446, 1424

Rein H., Tamayo D., 2015, Monthly Notices of the Royal Astronomical Society, 452, 376

Schödel R., et al., 2007, Astronomy \& Astrophysics, 469, 125

Tremaine S., Richstone D. O., Byun Y.-I., Dressler A., Faber S. M., Grillmair C., Koremndy J., Lauer T. R., 1994, The Astronomical Journal, 107, 634

Weinberg N. N., Milosavljević M., Ghez A. M., 2005, The Astrophysical Journal, 622, 878

Yelda S., Ghez A. M., Lu J. R., Do T., Meyer L., Morris M. R., Matthews K., 2014, The Astrophysical Journal, 783, 131

Yu Q., Tremaine S., 2003, The Astrophysical Journal, 599, 1129

Zwart S. F. P., McMillan S. L., 2002, The Astrophysical Journal, 576,899

Zwart S. F. P., Baumgardt H., McMillan S. L. W., Makino J., Hut P., Ebisuzaki T., 2006, The Astrophysical Journal, 641, 319

This paper has been typeset from a TEX/LATEX file prepared by the author. 